CARDIOVASCULAR MEDICINE

\title{
Sources of diagnostic inaccuracy of conventional versus new diagnostic criteria for myocardial infarction in an unselected UK population with suspected cardiac chest pain, and investigation of independent prognostic variables
}

\author{
J Trevelyan, E W A Needham, S C H Smith, R K Mattu
}

Heart 2003;89:1406-1410

\begin{abstract}
Objective: To assess the degree and sources of current diagnostic inaccuracy of serial conventional cardiac markers and ECGs compared with the new diagnostic criteria for myocardial infarction, with specific reference to physician specialty and the prognostic value of troponin $\mathrm{T}$.

Design: Prospective, blinded observational study.

Setting: University hospital.

Patients and interventions: All suspected cardiac chest pain admissions for six months, with additional blinded measurement of CK-MB mass and troponin T. World Health Organization and new criteria myocardial infarction diagnoses were made by an expert panel.

Main outcome measures: Diagnostic adjustment by expert panel; completeness of serial measurements; six months prognosis.

Results: A complete set of serial cardiac markers was not taken in $38.7 \%$ of patients, this being twice as likely when managed by non-cardiologists than by cardiologists $(p<0.0001)$. The WHO myocardial infarction diagnosis was adjusted by the expert panel in $4 \%$ of cases, this being $90 \%$ more likely in patients admitted under non-cardiologists $(p=0.026)$. The new criteria for myocardial infarction identified an additional $27.3 \%$ of infarcts, with a diagnostic alteration in $12.0 \%$ of the cohort; $45.2 \%$ of these cases had a potentially preventable cause for diagnostic adjustment. Only troponin T ( $p=0.0004$ ), ST depression $(p=0.003)$, and heart failure $(p=0.016)$ were independently predictive of prognosis.

Conclusions: Chest pain patients appear less likely to be fully and accurately assessed by non-cardiologists than by cardiologists. The new criteria for myocardial infarction identify $\sim 25 \%$ of additional patients as $\mathrm{MI}$, with potential additional advantages related to simplicity of diagnostic protocols. Troponin T was the most potent predictor of six month prognosis in an unselected cohort of chest pain admissions.
\end{abstract}

See end of article for authors' affiliations

Correspondence to: Dr R K Mattu, Department of Biological Sciences, University of Warwick, Coventry, CV4 7AL, UK; itrevelyan@hotmail.com

Accepted 31 March 2003
A recent consensus publication by the Joint European Society of Cardiology/American College of Cardiology committee ${ }^{1}$ proposed new criteria for the diagnosis of myocardial infarction. The document stresses the primacy of a rise in the cardiac specific isomers of the contractile proteins troponin $\mathrm{T}$ ( $\mathrm{cTnT}$ ) and troponin $\mathrm{I}$ in the diagnosis of myocardial infarction, replacing older, less reliable serum markers of myocardial damage. The previous gold standard, creatine kinase $\mathrm{MB}$ fraction (CK-MB) measured by mass assay (CK-MB mass), is recommended as an acceptable alternative. Despite the fact that these newer cardiac markers have been available for some time, the UK and other countries have continued to rely on serial ECGs and the conventional cardiac markers CK, aspartate transaminase (AST), and lactate dehydrogenase (LDH) in the first three days after the event for the diagnosis of myocardial infarction.

The troponins have potential advantages above and beyond improved diagnostic sensitivity and specificity, including simpler sampling protocols, a larger relative rise above normal limits, and a longer duration of elevation. In daily clinical practice these advantages may translate into important additional benefits when compared with diagnostic strategies based upon conventional markers.

Troponins also give prognostic information, though this is largely derived from selected cohorts with acute coronary syndromes, ${ }^{2-7}$ which have excluded patients with myocardial infarction, ${ }^{24}$ ST elevation/thrombolysis, ${ }^{357}$ and those without ECG changes. ${ }^{\circ}$
Reports suggest that patients managed by non-cardiologists have a worse outcome after acute coronary syndromes ${ }^{89}$ with underutilisation of the best pharmacological treatments and revascularisation possibly contributing to this. The National Service Framework for coronary heart disease in the UK has attempted to improve and standardise the quality and nature of care for all types of patients with coronary heart disease, and it stipulates that all high risk patients with acute coronary syndromes should be managed by a cardiologist. ${ }^{10}$ Despite this, in the UK and other countries these patients continue to be assessed and managed by noncardiologists. $^{8}{ }^{911} 12$ The advantages of the new criteria and serum markers for myocardial infarction may make evaluation of patients simpler and easier for busy clinical units. This should improve the diagnosis and appropriate targeting of treatments for patients presenting with suspected cardiac chest pain.

We therefore performed a prospective, blinded study to assess the degree and sources of current diagnostic inaccuracy in patients with suspected cardiac chest pain in a UK hospital using serial conventional cardiac markers and ECGs,

Abbreviations: AST, aspartate transaminase; $\mathrm{CK}$, creatine kinase; $\mathrm{CK}$ $M B$, creatine kinase isoenzyme $M B$; $c T n T$, cardiac troponin T; LDH, lactate dehydrogenase; MACE, major adverse cardiovascular events; UAP, unstable angina pectoris; WHO, World Health Organization 
with a blinded expert panel using the new diagnostic criteria for myocardial infarction for comparison. The specialist status of the attending physician was documented, and the care of the patient was free from interference or influence by the review panel. We also examined the impact of troponin $\mathrm{T}$ on the six months outcome in relation to major adverse cardiovascular events compared with other clinical and biochemical variables in our unselected cohort.

\section{METHODS \\ Patients}

All patients over the age of 18 years admitted with suspected cardiac chest pain to University Hospitals of Coventry and Warwickshire over a six month period were enrolled. The patients were divided into those admitted under a cardiologist (coronary care unit or specialist cardiology ward) or general physician (medical ward). Patient management by the admitting team was based upon conventional criteria using serial ECGs and daily CK/AST. Extra blood samples were drawn at 12 hours after symptom onset for the measurement of CTnT and CK-MB mass. At no stage was the admitting team aware of these results.

Following venesection, blood samples were stored at $4^{\circ} \mathrm{C}$ for 30 minutes, centrifuged at $3000 \mathrm{~g}$ for 10 minutes, and the serum stored at $-80^{\circ} \mathrm{C}$ before batch analysis. The serum concentration of myoglobin, CK-MB mass, and CTnT were measured quantitatively with the Elecsys 1010 machine, using nine minute STAT assays (Roche Diagnostics UK, Lewes, East Sussex, UK). The reference range for the cTnT assay in our laboratory was validated and underwent appropriate quality control against samples of known concentration. Our data were therefore analysed according to the manufacturer's recommended discriminator at that time, with an abnormal result defined as CTnT $>0.1 \mu \mathrm{g} / \mathrm{l}$.

Sensitivity of the cTnT test is $0.01 \mu \mathrm{g} / \mathrm{l}$. All analyses were done blind at a single laboratory, without knowledge of the patient's diagnosis. Coefficients of variation for the assays were: $\mathrm{CTnT}, 4.8 \%$ at $0.15 \mu \mathrm{g} / \mathrm{l}$ and $3.9 \%$ at $5.7 \mu \mathrm{g} / \mathrm{l}$; CK-MB mass, $4.0 \%$ at $6.3 \mu \mathrm{g} / \mathrm{l}$ and $4.0 \%$ at $55.8 \mu \mathrm{g} / \mathrm{l}$. Investigation of our CK-MB mass assay in a sample population of 1388 individuals generated median values of $0.97 \mu \mathrm{g} / \mathrm{l}$ in women and $1.35 \mu \mathrm{g} / \mathrm{l}$ in men, yielding 97.5 centile values of $2.88 \mu \mathrm{g} / \mathrm{l}$ and $4.94 \mu \mathrm{g} / \mathrm{l}$, respectively (manufacturer's data). The recommended discriminator value for the diagnosis of myocardial necrosis by this assay is therefore $5 \mu \mathrm{g} / \mathrm{l}$.

Standard 12 lead ECGs were obtained at presentation in all patients. Follow up ECGs were obtained daily, with additional ECGs if considered necessary by the physician in charge. ECG changes were categorised as follows: myocardial infarction (new Q waves or ST elevation $>1 \mathrm{~mm}$ in two limb leads or $>2 \mathrm{~mm}$ in two precordial leads), ischaemia (ST depression $>1 \mathrm{~mm}$ in two or more leads, T wave inversion, bundle branch block), other cardiac (for example, left ventricular hypertrophy, arrhythmia), or non-diagnostic/ normal.

\section{Diagnosis}

Following discharge, the patient's case notes were examined by a blinded, independent, expert panel comprising a data presenter (JT), a consultant cardiologist (RKM), a cardiology registrar, and a non-cardiologist. Three diagnoses were recorded. First, the "physician diagnosis", recorded on discharge by the physician in charge of the patient's care, was taken from the case notes. Second, the expert panel reached a "panel diagnosis" after reviewing the case notes, ECGs, and conventional cardiac markers. The physician and panel diagnoses were categorised as myocardial infarction, unstable angina pectoris (UAP), other cardiac (for example, stable angina, arrhythmia), or non-cardiac. The panel diagnosis of myocardial infarction was made by strict interpretation of the World Health Organization criteria, ${ }^{13}$ requiring two of the following three features: typical chest pain of $>30$ minutes' duration, serum conventional cardiac marker of more than twice the upper limit of normal, and definite ECG changes. Third, a "cTnT diagnosis" was made in accordance with the new recommended diagnostic criteria for myocardial infarction ${ }^{1}$, as follows: myocardial infarction was diagnosed if cTnT was $>0.1 \mu \mathrm{g} / \mathrm{l}$ with either typical chest pain, new Q waves, ECG indicating ischaemia (ST elevation or depression) myocardial infarction was excluded if cTnT was $<0.1 \mu \mathrm{g} / \mathrm{l}$.

The panel also examined whether the patient underwent a complete evaluation with respect to serial measurements over three days, and the influence of alterations in the patient's ward and attending physician.

\section{Prognosis}

Patients were monitored for six months after discharge from the index admission by examination of the case notes in all cases, in conjunction with telephone contact with the patient and the primary care physician if necessary. Follow up was $100 \%$ complete. All events were classified by an independent panel according to standard criteria for major adverse cardiovascular events (MACE): cardiac death, non-fatal myocardial infarction, percutaneous transluminal coronary angioplasty, coronary artery bypass grafting, or readmission with UAP.

\section{Statistics}

Categorical variables were analysed with $\chi^{2}$ Fisher's exact test and normally distributed continuous variables (age) with Student's $t$ test. Normality was tested with the KolmogorovSmirnov test. To assess the impact of clinical and biochemical markers on MACE-free survival, variables were entered in a Cox binary logistic regression analysis, using a forward regression technique. All analyses were done using SPSS version 10.0, and were two tailed, with $\mathrm{p}<0.05$ considered significant.

\section{Ethics}

The study was approved by the local research and ethics committee, and all subjects gave informed consent.

\section{RESULTS}

We enrolled 401 consecutive patients into the study, with a median time for presentation from symptom onset of 193 minutes. CK-MB mass and CTnT samples were drawn at a median time of 750 minutes after symptom onset, consistent with our predetermined protocol for collection after 12 hours. There were important demographic differences between patients admitted under a cardiologist compared with a medical physician (table 1): 235 (58.6\%) of the cohort were admitted to cardiology, and these patients were younger, $40 \%$ more likely to be male $(p=0.011), 50 \%$ more likely to be diabetic $(p=0.031), 110 \%$ more likely to be hypercholesterolaemic ( $p<0.0001)$, but $20 \%$ less likely to be hypertensive $(\mathrm{p}=0.015)$.

There were 155 patients (38.7\%) who did not have three full sets of conventional cardiac enzymes taken, with 137 (34.2\%) having an incomplete CK series and 136 (33.9\%) an incomplete AST series. However, 90 (22.4\%) of these had two complete sets of conventional markers, showing either a typical pattern of myocardial infarction within these $(\mathrm{n}=11)$ or having two negative sets $(\mathrm{n}=79)$, which is commonly, but sometimes mistakenly, accepted as excluding myocardial infarction. Among the 65 patients (16.2\%) with more significant gaps in conventional enzyme sampling, 45 $(11.2 \%)$ had two samples missing, with the remaining 
Table 1 Demographics by medical specialty

\begin{tabular}{|c|c|c|c|}
\hline & Whole cohort & Cardiology & Non-cardiology \\
\hline Number of patients & 401 & 235 & 166 \\
\hline Male & $259(64.6 \%)$ & $164(69.8 \%)$ & $95(57.2 \%)^{*}$ \\
\hline Age (median, range) & $68(29$ to 99$)$ & $66(29$ to 94$)$ & 68 (36 to 99) \\
\hline White & $362(90.3 \%)$ & $213(90.6 \%)$ & $149(89.8 \%)$ \\
\hline Diabetes & $68(17.0 \%)$ & $48(20.4 \%)$ & $20(12.0 \%) \dagger$ \\
\hline Hypercholesterolaemia & $182(45.4 \%)$ & $135(57.4 \%)$ & $47(28.3 \%) \ddagger$ \\
\hline Hypertension & $157(39.2 \%)$ & $81(34.5 \%)$ & $76(45.8 \%) \S$ \\
\hline Current or ex-smoker & $268(66.8 \%)$ & $163(78.0 \%)$ & $105(75.0 \%)$ \\
\hline Cerebrovascular disease & $37(9.2 \%)$ & $20(8.5 \%)$ & $17(10.2 \%)$ \\
\hline Previous myocardial infarction & $120(29.9 \%)$ & $74(31.5 \%)$ & $46(27.7 \%)$ \\
\hline Previous revascularisation & $64(16.0 \%)$ & $43(18.3 \%)$ & $21(12.7 \%)$ \\
\hline \multicolumn{4}{|c|}{$\begin{array}{l}\text { Values are } \mathrm{n}(\%) \\
\text { *RR } 1.4,95 \% \mathrm{Cl} 1.1 \text { to } 1.7 ; \mathrm{p}=0.011 \\
\text { tRR } 1.5,95 \% \mathrm{Cl} 1.0 \text { to } 2.2 ; \mathrm{p}=0.031 \\
\text { †RR } 2.1,95 \% \mathrm{Cl} 1.6 \text { to } 2.8 ; \mathrm{p}<0.0001 \\
\text { SRR } 08.95 \% \mathrm{Cl} 0.6 \text { to } 1.0 . \mathrm{p}=0.015\end{array}$} \\
\hline
\end{tabular}

missing either the second set $(n=12)$ or the first set $(\mathrm{n}=8)$. Twenty three of these 65 patients had been admitted to cardiology (9.2\% of admissions), and 42 (25.3\% of admissions) to a medical ward. Patients admitted to a medical ward were twice as likely to have an incomplete set of conventional cardiac markers taken than those admitted through cardiology (relative risk (RR), 2.0, 95\% confidence interval (CI) 1.6 to 2.6 ; p $<0.0001$ ).

The physician diagnosis and reclassified panel diagnosis are shown in table 2 . Re-examination of the case notes led to alteration in diagnosis among seven of the 121 patients discharged as myocardial infarction. A further nine patients were reclassified from non-infarction to infarction, yielding a significant diagnostic alteration in 16 of 401 cases $(4.0 \%)$. Examination of these diagnostic alterations with respect to specialty showed that reclassification occurred in five of 235 patients $(2.1 \%)$ admitted to cardiology, with four of these resulting from adjustment of the physician diagnosis of myocardial infarction at discharge to no myocardial infarction. In all four cases this was because the physician diagnosis of myocardial infarction had been made on elevation of cardiac markers to more than twice the baseline values but without reaching twice the diagnostic upper range. Strict interpretation of the WHO criteria for myocardial infarction required reclassification of these cases to the no infarction group, despite the fact that the recognised pattern may indeed reflect limited myocardial damage. Interestingly, all four of these cases were assigned a "cTnT diagnosis" of myocardial infarction by the panel using the new criteria.

In contrast, reclassification occurred in 11 of 166 patients (6.6\%) admitted through medical wards, both from myocardial infarction to no myocardial infarction and vice versa. These adjustments resulted from failure to recognise diagnostic elevations in cardiac markers $(n=8)$ or misinterpretation of non-diagnostic ECG changes (ST depression or T wave inversion, $\mathrm{n}=3$ ) in the presence of normal cardiac markers.
Patients admitted through medical wards were therefore $90 \%$ more likely to have the discharge diagnosis adjusted by the panel (RR $1.9,95 \%$ CI 1.3 to $2.7 ; p=0.026$ ).

Table 3 shows the impact of implementing the new diagnostic criteria upon the physician diagnosis. Forty four of 161 myocardial infarction cases $(27.3 \%)$ diagnosed by the new criteria had been discharged as not myocardial infarction. These 44 cases comprised $33.8 \%$ of those discharged as UAP, $15.2 \%$ of those discharged as "other cardiac", and 7.0\% of those classified as "non-cardiac". Additionally, the physician diagnosis of myocardial infarction was rejected in four cases $(3.3 \%)$ where elevation of conventional markers was from non-cardiac sources. Overall, a significant diagnostic alteration occurred in 48 of 401 patients (12.0\%) when applying the new criteria. Further examination of these 48 cases (table 4) shows that in $26(54.2 \%)$ the diagnostic adjustment was because of inaccuracy of the conventional cardiac markers, while in 22 cases $(45.8 \%)$ the cause may have been preventable. Fourteen $(29.2 \%)$ of these cases did not have a complete set of conventional markers for analysis, while eight cases $(16.7 \%)$ were related to inappropriate interpretation of data by the physician in charge.

Table 3 Physician diagnosis and reclassification according to the new diagnostic criteria for myocardial infarction

\begin{tabular}{lll}
\hline & \multicolumn{2}{l}{$c T n T$ diagnosis } \\
\cline { 2 - 3 } Physician diagnosis & $M I(n=161)$ & No $M I(n=240)$ \\
\hline$M I(n=121)$ & $117(96.7 \%)$ & $4(3.3 \%)$ \\
UAP $(n=71)$ & $24(33.8 \%)$ & $47(66.2 \%)$ \\
Other cardiac $(n=66) 10(15.2 \%)$ & $56(84.8 \%)$ \\
Non-cardiac $(n=143)$ & $10(7.0 \%)$ & $133(93.0 \%)$ \\
\hline \multicolumn{2}{l}{ Values are $n(\%)}$.
\end{tabular}

Table 2 Physician diagnosis and retrospective adjustment by expert panel

\begin{tabular}{lllll}
\hline \multicolumn{5}{c}{ Panel diagnosis } \\
\cline { 2 - 5 } Physician diagnosis & MI $(\mathbf{n}=123)$ & UAP $(\mathbf{n}=\mathbf{7 7})$ & $\begin{array}{l}\text { Other cardiac } \\
(\mathbf{n}=64)\end{array}$ & $\begin{array}{l}\text { Non-cardiac } \\
(\mathbf{n}=137)\end{array}$ \\
\hline MI $(n=121)$ & $114(94.2 \%)$ & $6(5.0 \%)$ & $1(0.8 \%)$ & 0 \\
UAP $(n=71)$ & 0 & $71(100 \%)$ & 0 & 0 \\
Other cardiac $(n=66)$ & $3(4.5 \%)$ & 0 & $63(95.5 \%)$ & 0 \\
Non-cardiac $(n=143)$ & $6(4.2 \%)$ & 0 & 0 & $137(95.8 \%)$ \\
\hline Values are $n(\%)$. & & & \\
MI, myocardial infarction; UAP, unstable angina pectoris. & &
\end{tabular}


Table 4 Sources of diagnostic adjustment by new criteria for myocardial infarction

\begin{tabular}{|c|c|c|c|}
\hline $\begin{array}{l}\text { Potential source } \\
\text { of diagnostic } \\
\text { adjustment }\end{array}$ & $\begin{array}{l}\text { Total } \\
(n=48)\end{array}$ & $\begin{array}{l}\text { Ward } \\
\text { adjustment } \\
\text { days } 1-3\end{array}$ & $\begin{array}{l}\text { Physician } \\
\text { adjustment days } \\
1-3\end{array}$ \\
\hline $\begin{array}{l}\text { Incomplete serial } \\
\text { cardiac markers }\end{array}$ & $14(29.2 \%)$ & $4(28.6 \%)$ & $10(71.4 \%)$ \\
\hline $\begin{array}{l}\text { Inappropriate } \\
\text { physician diagnosis }\end{array}$ & $8(16.7 \%)$ & $2(25.0 \%)$ & $4(50.0 \%)$ \\
\hline $\begin{array}{l}\text { Sensitivity/ } \\
\text { specificity of } \\
\text { conventional } \\
\text { markers }\end{array}$ & $26(54.2 \%)$ & $10(38.5 \%)$ & $18(69.2 \%)$ \\
\hline
\end{tabular}

These 48 cases who underwent diagnostic adjustment by the new criteria were subsequently studied for any correlation between potential causes of diagnostic adjustment with either human error or issues related to assay sensitivity/ specificity. We evaluated two suspected causes of human error that commonly occur-namely, a change in the patient's ward and an alteration in the physician in charge during the first three days (table 4). Examination of these potentially avoidable factors shows that these occurred with similar frequency where marker sensitivity/specificity was to blame and where there might have been human error. While this does not prove that such factors are not causative, they do not seem to occur more often in those cases with a potentially avoidable reason for diagnostic inaccuracy.

The six month composite end point of any MACE occurred in 61 patients $(15.2 \%)$. To determine if cTnT provided prognostic information beyond accepted clinical markers of high risk (age, sex, diabetes, ST depression $>1 \mathrm{~mm}$ on the ECG, presence of heart failure, and post-myocardial infarction defined by WHO criteria) ${ }^{14}$ these variables, together with CK-MB mass, were entered in a Cox multiple logistic regression analysis (table 5). When CK-MB mass of $>5 \mu \mathrm{g} / \mathrm{l}$ alone is entered as the biochemical risk marker (model 1 ), it is strongly predictive of MACE outcome $(p=0.002)$, together with ST depression $>1 \mathrm{~mm}(\mathrm{p}=0.001)$ and the presence of heart failure $(p=0.018)$. In contrast, when cTnT is added to the analysis (model 2), CK-MB mass loses this independent predictive value, with $\mathrm{cTnT}>0.1 \mu \mathrm{g} / \mathrm{l}$ $(\mathrm{p}=0.0004)$, ST depression $>1 \mathrm{~mm}(\mathrm{p}=0.003)$, and the presence of heart failure $(p=0.016)$ emerging as the independent variables. Thus CK-MB mass appears to track the prognostic ability of CTnT, albeit as a less potent risk stratifier, and is not independently predictive when entered in a model with cTnT. Finally, we evaluated the status of the physician in charge of the patient as a clinical risk marker (model 3). We found that consultant specialty did not independently influence patient prognosis, and had no impact on the other risk variables in this model, although it was noted that cardiologists care for the higher risk cardiac patients (data not shown).

\section{DISCUSSION}

In an unselected cohort of patients with suspected cardiac chest pain attending a UK hospital, $27.3 \%$ of myocardial infarcts (44/161) diagnosed by the new criteria using cTnT are missed by physicians employing the current diagnostic strategies of serial CK/AST and ECGs. The current strategy also resulted in $3.3 \%$ of myocardial infarcts (4/121) being incorrectly assigned this diagnosis. Over half these diagnostic alterations $(54.2 \%, 26 / 48)$ appeared to be caused by lack of sensitivity/specificity of the conventional markers. However, in $45.8 \%$ of cases $(22 / 48)$, the diagnostic inaccuracy was found to be potentially avoidable and was related to inappropriate interpretation of diagnostic results and failure to complete the current three day sampling strategy. Indeed, $38.7 \%$ of the cohort (155/401) did not undergo a full evaluation with serial conventional cardiac markers.

In our cohort, we have found that patients cared for by a cardiologist are consistently more likely to be assessed with a full set of conventional cardiac markers and to have an accurate diagnosis made by the physician responsible for patient care. The cardiology cases where there was a conflict of diagnoses between our panel and the physician were predominantly "grey" cases in which the correct diagnosis was unclear. In contrast, patients admitted through medical wards were more likely to have an incomplete series of conventional markers taken, and $6.6 \%$ of cases (11/166) were discharged with an incorrect diagnosis owing to misinterpretation of available data. The underlying causes are unclear, but inadequate levels of staffing, training, or supervision of junior staff may contribute, although we did not evaluate these factors. We suspected that patient relocation between wards and between consultant teams could be responsible, but were unable to demonstrate any clear relation with diagnostic alteration. While we have not tested whether the introduction of the new diagnostic criteria with simpler sampling requirements would have resolved these issues, the adoption of more straightforward and rapid diagnostic protocols may assist clinicians in reaching more certain diagnoses.

Previous studies have shown that in selected cohorts with acute coronary syndromes, the cardiac troponins aid distinction of high and low risk individuals. ${ }^{2-7}{ }^{15}$ We found that troponin $\mathrm{T}$ provided prognostic information superior to clinical and other biochemical variables in our unselected cohort with suspected cardiac chest pain, and support the earlier observations in other acute coronary syndromes. CKMB mass provides similar prognostic prediction, but appears to track with cTnT as it offers no independent prognostic information when cTnT is used. The ability of the new markers to distinguish reliably the $3.3 \%$ of myocardial

Table 5 Multiple logistic regression models for major adverse cardiovascular events

\begin{tabular}{|c|c|c|c|c|c|c|}
\hline \multirow[b]{2}{*}{ Risk variable } & \multicolumn{2}{|l|}{ Model 1} & \multicolumn{2}{|l|}{ Model 2} & \multicolumn{2}{|l|}{ Model 3} \\
\hline & Coefficient value & $p$ Value & Coefficient value & $p$ Value & Coefficient value & p Value \\
\hline Age & 0.137 & 0.71 & 0.187 & 0.67 & 0.278 & 0.60 \\
\hline Sex & 0.086 & 0.77 & 0.009 & 0.92 & 0.120 & 0.73 \\
\hline Diabetes mellitus & 0.516 & 0.47 & 0.396 & 0.53 & 0.175 & 0.68 \\
\hline WHO MI & 0.008 & 0.93 & 0.189 & 0.66 & 0.131 & 0.72 \\
\hline ST depression $>1 \mathrm{~mm}$ & -1.088 & 0.001 & -0.983 & 0.003 & 1.077 & 0.003 \\
\hline Heart failure & -0.752 & 0.018 & -0.763 & 0.016 & 0.934 & 0.017 \\
\hline CK-MB $>5 \mu \mathrm{g} / \mathrm{I}$ & 0.925 & 0.002 & 0.046 & 0.83 & 0.029 & 0.87 \\
\hline $\mathrm{cTnT}>0.1 \mu \mathrm{g} / \mathrm{l}$ & & & -1.119 & 0.0004 & 1.26 & 0.0003 \\
\hline Consultant specialty & & & & & 0.141 & 0.71 \\
\hline
\end{tabular}

CK-MB, creatine kinase MB isoenzyme; $c T n T$, cardiac troponin $T ; M I$, myocardial infarction. 
infarcts incorrectly diagnosed by CK/AST elevations of nonmyocardial origin will help avoid psychological, social, and physical detriment to these patients and their families, and inappropriate use of health care resources.

The new diagnostic criteria for myocardial infarction have an important impact among patients admitted with suspected cardiac chest pain, and consideration should be given to the adoption of these guidelines. The limitations of current diagnostic strategies are not only related to performance of the markers themselves, but also to the time consuming nature of the associated diagnostic protocols, which are subject to more opportunities for human error in data collection. The introduction of simpler diagnostic strategies using more sensitive and specific markers should allow more accurate diagnosis, provide prognostic value beyond that afforded by current risk factors, and may also shorten the hospital stay. Our observed differences in management between general medical and cardiological care support the recommendations of the National Service Framework for assessment of the majority of these patients in a specialist setting.

\section{ACKNOWLEDGEMENTS}

We gratefully acknowledge W-K Lee for help with data collection and database entry, M Stokes for sample analysis, cardiac nurse practitioners S Hobday and C Willmott, and all junior doctors at Walsgrave Hospital who helped with sample collection. JT is supported by the National Heart Research Fund and RKM and EWAN by the British Heart Foundation.

\section{Authors' affiliations}

J Trevelyan, R K Mattu, Department of Cardiology, University Hospitals of Coventry and Warwickshire NHS Trust, Coventry, UK

S C H Smith, Department of Clinical Biochemistry, University Hospitals of Coventry and Warwickshire NHS Trust

E W A Needham, Department of Biological Sciences, University of Warwick, Coventry

\section{REFERENCES}

1 The Joint European Society of Cardiology/American College of Cardiology Committee. Myocardial infarction redefined - a consensus document of the joint European Society of Cardiology/American College of Cardiology committee for the redefinition of myocardial infarction. Eur Heart $J$ 2000;21:1502-13.

2 Hamm CW, Ravkilde J, Gerhardt W, et al. The prognostic value of serum troponin T in unstable angina. N Engl J Med 1992;327:146-50.

3 Hamm CW, Goldmann BU, Heeschen C, et al. Emergency room triage of patients with acute chest pain by means of rapid testing for cardiac troponin $T$ or troponin I. N Engl J Med 1997;337:1648-53.

4 Ravkilde J, Nissen H, Horder M, et al. Independent prognostic value of serum creatine kinase isoenzyme $M B$ mass, cardiac troponin $T$ and myosin light chain levels in suspected acute myocardial infarction. Analysis of 28 months of follow-up in 196 patients. J Am Coll Cardiol 1995;25:574-81.

5 Lindahl B, Venge P, Wallentin L. Relation between troponin T and the risk of subsequent cardiac events in unstable coronary artery disease. The FRISC study group. Circulation 1996;93:1651-7.

6 Ohman EM, Armstrong PW, Christenson RH, et al. Cardiac troponin T levels for risk stratification in acute myocardial ischemia. GUSTO IIA investigators. N Engl J Med 1996;335:1333-41.

7 Antman EM, Tanasijevic M, Thompson B, et al. Cardiac-specific troponin I levels to predict the risk of mortality in patients with acute coronary syndromes. N Engl J Med 1996;335:1342-9.

8 Schreiber TL, Elkhatib A, Grines CL, et al. Cardiologist versus internist management of patients with unstable angina: treatment patterns and outcomes. J Am Coll Cardiol 1995;26:577-82.

9 Jollis JG, DeLong ER, Peterson ED, et al. Outcome of acute myocardial infarction according to the specialty of the admitting physician. N Engl J Med 1996:335:1880-7

10 Department of Health. The National Service Framework for coronary heart disease. London: Department of Health, 2000.

11 Drory Y, Shapira I, Goldbourt U, et al. Emergency room referral to internal medicine wards or to coronary care units of patients with first acute myocardial infarction. Israel study group on first acute myocardial infarction. $J$ Med 2000;31:90-100.

12 Tu JV, Austin PC, Chan BT. Relationship between annual volume of patients treated by admitting physician and mortality after acute myocardial infarction. JAMA 2001;285:3116-22.

13 Tunstall-Pedoe H, Kuulasmaa K, Amouyel P, et al. Myocardial infarction and coronary deaths in the World Health Organization MONICA project. Circulation 1994;90:583-612.

14 Calvin JE, Klein LW, VandenBerg EJ, et al. Validated risk stratification mode accurately predicts low risk in patients with unstable angina. J Am Coll Cardiol 2000;36:1803-8.

15 Ravkilde J, Horder M, Gerhardt W, et al. Diagnostic performance and prognostic value of serum troponin $T$ in suspected acute myocardial infarction. Scand J Clin Lab Invest 1993;53:677-85.

\section{FROM BMJ JOURNALS}

\section{Defining clinical features of amiodarone induced optic neuropathy}

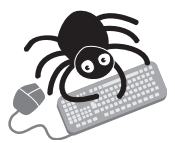

Please visit the Heart website [www. heartjinl. com] for a link to the full text of this article. miodarone is known to cause optic neuropathy that can result in visual loss. It may 1 present similarly to that of non-arteritic anterior optic neuropathy (NAION). Three Icases are described in an attempt to distinguish the conditions.

All three patients (aged 59-72) presented with mild visual loss and bilateral optic disc swelling. Two had unilateral subjective visual complaints while one was asymptomatic. All had been taking 200mg of amiodarone daily for 3-12 months. Visual acuity improved in all after discontinuing the drug and their dyschromatopsia and visual field defects resolved. In general optic disc swelling lasted for more than six months, a finding which would be unusual in NAION. Swelling lasted up to four months after discontinuing the drug.

Compared with NAION, amiodarone induced neuropathy has an insidious onset, less visual loss, longer duration of disc oedema and is more commonly bilateral. The diagnosis can be confirmed only by observing slow resolution after stopping the intensely cationic amphophilic medication which accumulates by interaction with polar lipids, allowing it to be deposited as lysosomal inclusion bodies in the optic nerve.

The authors suggest periodic ophthalmic screening could be useful in patients taking amiodarone (who have a risk of optic neuropathy of $1.79 \%$ in one series), while visual symptoms should provoke expeditious fundoscopy.

Other ocular side effects previously reported with amiodorone include corneal microdeposits, anterior subcapsular lens opacities, multiple chalazia and dry eye syndrome.

A British Journal of Ophthalmology 2003;87:420-422. 